\title{
Integrating equity into environmental health practice: findings of a pilot study
}

\author{
Karen Rideout ${ }^{*, \dagger}$ and Dianne Oickle* \\ *BC Centre for Disease Control, Vancouver, BC, Canada. \\ ${ }^{\dagger}$ National Collaborating Centre for Environmental Health, Vancouver, BC, Canada. \\ *National Collaborating Centre for Determinants of Health, Antigonish, NS, Canada.
}

\begin{abstract}
The existence of health inequities is a priority public health issue in Canada, but it is not clear how - or how well - equity is embedded into environmental health practice. To assess how health equity and the social determinants of health intersect with their practice, we conducted two focus groups with public health inspectors in British Columbia and Nova Scotia. We explored where social barriers affect compliance, how practitioners respond, and where there are resource or knowledge gaps that limit practitioner response. Participants identified barriers related to socioeconomic status, culture, education, and geography. They responded to these barriers largely in ad hoc ways, using makeshift or borrowed tools, personal collaboration networks, and communication strategies, and by exercising discretion and progressive enforcement. Public health inspectors indicated they were motivated to take action but felt uncertain of their scope and role in doing so. Findings indicate that health equity does relate to environmental health practice and that there is a need for additional resources, organizational supports from management, and further research to identify and evaluate best practices.
\end{abstract}

Key words: environmental health, health equity, public health practice, social determinants of health

Health inequities are health differences associated with social disadvantages that are modifiable and considered unfair (National Collaborating Centre for Determinants of Health, 2015). Inequities can lead to differences in environmental exposures, vulnerabilities to and outcomes from those exposures, and access to services (Blas and Kurup, 2010). Although health equity is increasingly referenced in policy frameworks as a priority for public health practitioners (British Columbia Ministry of Health, 2013; Nova Scotia Department of Health and Wellness, n.d.), it is not known how, or how deeply, these principles are embedded into practice. This study explored how health equity and the social determinants of health (SDH) intersect with environmental health regulatory practice.

Disparities in income, language, or education could create barriers to compliance with some environmental health regulations. The way public health inspectors (PHIs) respond in the presence of such barriers has the potential to mitigate or intensify the inequities created by those barriers - particularly if PHIs are unaware of how social disadvantage creates compliance challenges. There may be opportunities for PHIs to facilitate sustained behavior change or advocate for more responsive regulations by understanding the barriers posed by inequities. However, we know little about the knowledge and capacity of

Corresponding author: Karen Rideout (email: karen.rideout@ bccdc.ca)
PHIs to identify and address health inequities. A British Columbia (BC) study of health promotion practices suggested that practitioners there felt ill-equipped to address social issues in their work (Campbell et al., 2011). Ontario PHIs doing housing inspections perceived issues of vulnerability as beyond the scope of their role as defined by legislative or health unit policy (Lefebvre et al., 2012). Given the potential impacts on practice, there is a need to better articulate the role of PHIs with respect to the SDH. This study aimed to characterize how health equity relates to environmental health regulatory practice, identify ways that practitioners respond when equityrelated barriers are present, and explore how to integrate health equity into practice more explicitly.

\section{Methods}

We used focus group methodology because of its ability to identify relevant topics, appropriate terminology, and perspectives from a group of people (Kvale, 1996; The Health Communication Unit, 2002). Two focus groups were held with PHIs in Halifax, Nova Scotia (NS), and Surrey, BC, in March 2014. Although not intended to represent all regions of Canada, the study sites reflect a range of community and organizational characteristics. Additional details about recruitment and study design are reported elsewhere (Rideout and National Collaborating Centre for Determinants of Health, 2015). The research 
was approved by the University of British Columbia Behavioural Research Ethics Board and the Fraser Health Authority Research Ethics Board.

Focus group moderators queried PHIs about vulnerable populations, barriers that affect compliance, PHI responses, and options to integrate health equity considerations into their practice. They avoided the terms "equity" and "social determinants of health" to circumvent any preconceptions about what those terms mean and to allow exploration of issues that participants did not initially relate to equity.

Both authors conducted thematic analysis of the focus group transcripts using QSR NVivo $10^{\circledR}$. We developed initial codes based on the project objectives and adapted them as themes emerged from the data (LeCompte and Schensul, 1999; Rubin and Rubin, 2005). D.O. analysed both focus group transcripts; K.R. verified the coded transcripts for accuracy and added additional codes.

\section{Results}

\section{Participants}

The focus groups explored participants' professional experiences as PHIs in their current and previous jobs. Eleven participants from NS and $\mathrm{BC}$ brought a range of experience and expertise. Three male and three female PHIs from NS included food safety specialists and inspector specialists who worked in food premises inspections, food safety training, water and waste, occupational health and safety, and environmental protection. One male and four female BC participants were from a mix of urban, suburban, and rural communities southeast of Vancouver and dealt with communicable disease, drinking water, food premises, personal services establishments, and recreational water.

There were few notable differences between the two focus groups. The BC PHIs spoke more about cultural barriers faced by new immigrants, reflecting the high immigration rate in the region. In NS, where PHIs work out of several governmental departments rather than a regionalized public health system, the group discussed jurisdictional challenges with environmental health programs that are spread across government departments with different priorities. It was difficult to know whether this reflected regional differences or if the discussions simply followed different trajectories. As such, we report the data collectively.

\section{Health equity and environmental health practice}

Focus group participants identified equity-related factors that presented barriers to compliance for some business or facilities operators. These related to socioeconomic status, cultural differences, language and literacy, and geographic location (Table 1).

Some operators had difficulty paying for infrastructure upkeep and equipment repairs, and some employees were challenged by low wages and precarious employment. PHIs
Table 1. Barriers to compliance with environmental health regulations that reflect a need for equity-integrated practice

\begin{tabular}{|l|l|}
\hline Social factor & Barriers \\
\hline $\begin{array}{l}\text { Socioeconomic } \\
\text { status }\end{array}$ & $\begin{array}{l}\text { Cash flow for repairs, maintenance, } \\
\text { or monitoring } \\
\text { Income and job security }\end{array}$ \\
\hline Culture & $\begin{array}{l}\text { Lack of English language skills } \\
\text { Understanding of PHI role }\end{array}$ \\
\hline $\begin{array}{l}\text { Language and } \\
\text { literacy }\end{array}$ & $\begin{array}{l}\text { Low English language literacy } \\
\text { Low computer and web literacy }\end{array}$ \\
\hline $\begin{array}{l}\text { Geographic } \\
\text { location }\end{array}$ & $\begin{array}{l}\text { Slow access to parts, labour, and } \\
\text { expertise } \\
\text { High cost to transport parts and } \\
\text { equipment or to access maintenance } \\
\text { personnel } \\
\text { Lack of approved food sources that } \\
\text { meet food safety regulations }\end{array}$ \\
\hline
\end{tabular}

observed workers without paid sick leave handling food even when they had symptomatic gastrointestinal illness:

There are employers that I've deal with in the past... that say, if you can't find somebody to cover your shift, you have to come into work or you're fired... And they're sneezing... or [have vomiting and diarrhea] and trying to hold it together. [But] they come into work.

Operators of food premises or private drinking water systems, particularly small businesses and those in small communities, often lacked sufficient funds to make timely repairs or follow required monitoring and upkeep procedures. Participants observed that stress experienced by the operators sometimes intensified the effects of financial barriers. Some PHIs noticed that business or facilities operators experiencing personal or family issues became less compliant until those issues were resolved.

Participants discussed challenges faced by operators with different cultural backgrounds. Business owners not accustomed to inspectors with an educational role can be hesitant to admit difficulties or ask for guidance from the PHI.

[The interpreter] came down and talked to him about the way [the system here] works to assist the operator in getting to compliance, and not the other way around. And then they sort of start to understand that when I phone, I'm not trying to make trouble for them.

They may view the PHI as someone who might shut down their business, but not as someone who could help them reach compliance. Some food premises operators used traditional food practices for which there was no local food safety guidance. This raised compliance concerns that were further complicated if language barriers prevented clear communication about processes and food safety protocols.

Limited English language skills created additional barriers. Simple instructions may be manageable, but the intricacies of why health regulations are important and how to put them into practice were lost without shared language. 
It's one thing to tell them to fix the dishwasher, it's another thing to [help non English speakers] understand how the thing actually works.

Low literacy levels had similar effects regardless of language. People with limited reading skills might not fully comprehend written information. Because many people try to hide their literacy challenges, this barrier can be difficult to identify.

Increased time and cost to access parts, skilled labour, and expertise in small or remote communities can prohibit timely maintenance and repairs to equipment.

Pretty much everything has to come [from the city], so when you're [in a small place], it's longer to get things ordered, longer to get repair people, it's harder to find repair people to fix certain dishwashers.

PHIs reported making judgments about public health risk when deciding whether operations could continue while waiting for repairs. Participants who had worked in remote communities also noted a challenge where approved sources of food were not readily available and wild-caught foods were served.

In a lot of communities, that's what they lived on, and they couldn't get access to any other kind of protein ... so I would make it the safest possible for them, instead of saying "no, you can't have it at all."

\section{Responding to equity-related barriers}

Where participants recognized SDH-related barriers, they responded in a variety of ad hoc ways (Table 2). They expressed interest and desire to take action on observed inequities, but felt their ability to respond was constrained by limited access to tools, resources, and organizational supports.

The PHIs in this study expressed frustration with insufficient resources to respond to SDH-related barriers and employed creative strategies to overcome those challenges. They enlisted relatives or neighbours as translators, used multilingual print resources from other jurisdictions, spoke more simply, and even "acted out" instructions. Although not considered ideal or effective for communicating complex information, these were their best available options.

Table 2. Strategies used by PHIs in response to equity- and SDH-related barriers

\begin{tabular}{|l|l|}
\hline Strategies & Examples \\
\hline Communication & $\begin{array}{l}\text { Borrowing multilingual print resources } \\
\text { Using family as translators } \\
\text { Demonstrating instructions }\end{array}$ \\
\hline Relationships & $\begin{array}{l}\text { Building trust } \\
\text { Avoiding heavy-handed enforcement }\end{array}$ \\
\hline Discretion & $\begin{array}{l}\text { Progressive enforcement techniques } \\
\text { Prioritizing risks and setting achievable } \\
\text { goals } \\
\text { Considering context }\end{array}$ \\
\hline Collaboration & $\begin{array}{l}\text { Referring to supports or agencies outside } \\
\text { health protection }\end{array}$ \\
\hline
\end{tabular}

When PHIs recognized that barriers beyond someone's control were preventing compliance, they relied on relationshipbuilding and communication strategies to encourage operators to share their challenges and allow the PHI to help when possible.

They really like... having that relationship with an inspector ... so they feel comfortable in telling them. . . why they can't get this done or why things are going wrong. Or they'll call and say what can we do about this?

PHIs described building trust rather than using enforcement as a strategy to address health protection concerns.

I'm not going to throw you under the bus... This is, in the long run,

... what needs to be done, and I will work with you.

Participants often used discretion and progressive enforcement (i.e., gradual implementation of corrective action) when barriers were present. They prioritized issues according to risk and tried to set achievable goals that still protected public health.

It's really important not to overuse [legislation] — not to not use it, but it's really important not to use it wrongly. And that's where our discretion comes in. . Not being heavy-handed, but making sure that we are listening to the public's health. That is paramount.

PHIs sometimes looked outside the health system to link individuals with supports or services. They reported finding funding to pay for equipment upgrades or referring to social service agencies, relying on their own networks to identify supports. Challenges to such collaboration are explored in the next section.

\section{Integrating health equity in practice}

Operational challenges impacted participants' ability to respond when they recognized barriers related to the SDH. High workloads and job stress made it difficult for them to invest time to build relationships. PHIs often used their own time to seek out resources or refer clients. They felt that a lack of collaborative systems hindered referrals, and they were not always clear of their jurisdiction to act in a given context.

All of us have the same certification, but I [PHI not responsible for food premises] could go to a market and see things. What are we supposed to do with this? Am I supposed to call the manager or call an inspector? Am I overstepping my bounds this way? I'm still a health inspector, this is still a health hazard, but the system is... fragmented.

Some PHIs reported a lack of support from their superiors and some were unsure how much discretion they could use. They found it difficult to balance hazards where enforcement of one regulation might create another public health hazard. PHIs noted that prescriptive policies sometimes forced them to enforce rules rather than address the underlying issues that create public health risks. 


\section{Discussion}

\section{Intersections of health equity and the SDH with environmental health practice}

This pilot study confirms that health equity intersects with environmental health practice in multiple ways. While the term "health equity" was not raised, the discussions clearly identified a relationship between the SDH and PHI practice. Participants recognized barriers related to socioeconomic status, communication, education, and geography that made it difficult for some people to comply with health protection regulations. Language, education, and multiple personal barriers have also been identified as challenges to food safety regulation in Ontario (Pham et al., 2010) and to health promotion activities in BC (Campbell et al., 2011). Participants also recognized the impact of these $\mathrm{SDH}$ on their own ability to do their job effectively.

\section{Ad hoc practitioner responses to perceived health inequities}

PHIs in this study expressed strong interest in actively addressing the $\mathrm{SDH}$, but without specific guidance, they responded in ad hoc ways. They relied heavily on communication and relationships to help operators facing SDH-related barriers. They felt that compliance was more achievable when they considered the individual context, prioritized corrective actions, engaged in open communication, and built trust with operators than if they used a more authoritarian approach. This sentiment is expressed elsewhere in relation to housing inspections (Lefebvre et al., 2012). PHIs in this study described frustration that they could not respond adequately due to a lack of supports and tools. They expressed desire for their profession to be better recognized as capable to address the SDH through their work:

We have a broad understanding. We can assess risk really well, and we apply it through food, water, air, built environments, everything.

These findings indicate openness to an equity-integrated environmental health practice.

\section{Toward an equity-integrated environmental health practice}

These results highlight a need to better support PHI action on health equity. Practitioners need tools and resources that fit their practice, organizational structures that facilitate communication and collaboration, and guidance on how to respond to SDH-related barriers. Further research would shed light on how PHIs can and should practice with an equity lens.

The ad hoc use of language tools suggests that PHIs need access to resources to work around SDH-related barriers. Although real-time interpreter services are not practically or financially feasible, multilingual print resources could be adapted for use in other areas without excessive costs, a need echoed by PHIs in Ontario (Lefebvre et al., 2012). Training in cultural competency may also help PHIs work across cultural and language barriers.

PHI action on health equity would benefit from networking and collaboration support. These findings indicate that PHIs rely on colleagues for support and learning, particularly for dealing with the kind of complex issues that arise due to inequities. Formalized collaboration with other branches of public health would support this internal networking. Collaborations with external agencies such as social services, housing, and community organizations would facilitate referrals that support vulnerable people to leverage the collective skills, mandates, and authority of a variety of agencies (Nova Scotia Department of Health and Wellness, n.d.). Inter-agency networks (Lefebvre et al., 2012), as well as ongoing relationships with government and individuals, could enable PHIs to respond to inequities (British Columbia Ministry of Health, 2013). Environmental health professionals in the United Kingdom were provided with SDH training and authority to require housing managers to improve living conditions. Although the outcomes continue to be evaluated, combining health equity training with regulatory authority shows promise for protecting health (Stewart and Bourn, 2013).

Clear articulation of roles and responsibilities would support PHIs to recognize and respond to health inequities. PHIs can use discretionary enforcement and locally adapted policies to both limit the impact of social barriers and encourage operators to take prospective - rather than corrective - action to control health risks (Lefebvre et al., 2012). However, participants reported feeling unsure about the extent to which they could use discretionary enforcement and whether their responses to social barriers overstepped their professional boundaries. They need clear guidance and support to use such approaches.

It will be important to build on this exploratory research to further assess PHI knowledge and to identify resource and training needs that could support integration of equity into practice. Canada-wide research would highlight provincial or regional differences in the types of barriers faced, the strategies used to address them, and the influence of different organizational structures. Evaluations of current ad hoc responses would facilitate consistent application of the most effective strategies.

\section{Conclusion}

Public health has broadened its scope to incorporate health equity and social and ecological determinants of health (Canadian Public Health Association, 2015). Public health agencies in Canada increasingly mandate public health professionals to engage in health promotion and contribute to improving health equity (British Columbia Ministry of Health, 2013; Health Officers Council of British Columbia, 2013; Nova Scotia Department of Health and Wellness, n.d.; Public Health Agency of Canada, 2008). Integrating SDH and health equity into professional competencies for PHIs would reinforce the knowledge and skills necessary to support a health equity role for PHIs. They will be better equipped to recognize and respond to operators who are facing equity-related barriers to compliance, including those who may face hidden barriers. If PHIs can address those barriers, the need for enforcement 
could decrease. Recognizing the impact of the SDH on operators' abilities to comply with health protection regulations and supporting PHI responses are essential to operationalize provincial and national health equity goals.

\section{Acknowledgements}

The authors wish to acknowledge Ken Shaw from the Fraser Health Authority and Gary O'Toole from the Nova Scotia Department of Health and Wellness for assistance with design and recruitment of the focus groups. We also wish to acknowledge Hannah Moffatt and Sylvia Struck for their work in the early phases of planning and implementation for this project, and Tom Kosatsky and Connie Clement for valuable input on the content and direction of the project. Funding for the National Collaborating Centre for Determinants of Health and the National Collaborating Centre for Environmental Health is provided by the Public Health Agency of Canada. The views expressed herein do not necessarily represent the views of the Public Health Agency of Canada.

\section{References}

Blas, E. and Kurup, A. S. (Eds.). 2010. Equity, social determinants and public health programmes. Geneva, Switzerland: World Health Organization.

British Columbia Ministry of Health. 2013. Promote, protect, prevent: Our health begins here. BC's guiding framework for public health. Victoria, BC. Available at http://www.health.gov.bc. ca/library/publications/year/2013/BC-guiding-framework-for-publichealth.pdf. (Accessed 26 February 2016.)

Campbell, A. C., Foggin, T. M., Elliott, C. T. and Kosatsky, T. 2011. Health promotion as practiced by public health inspectors: The BC experience. Can. J. Public Health. 102(6): 432-436. doi: 10.17269/cjph.102.2751.

Canadian Public Health Association. 2015. Global change and public health: Addressing the ecological determinants of health. Ottawa, ON. Available at http://www.cpha.ca/uploads/policy/edh-discussion_ e.pdf. (Accessed 26 February 2016.)

Health Officers Council of British Columbia. 2013. Health inequities in British Columbia. North Vancouver, BC. Available at http:// healthofficerscouncil.files.wordpress.com/2012/12/health-inequitiesin-bc-april-15-2013.pdf. (Accessed 26 February 2016.)
Kvale, S. 1996. InterViews: An introduction to qualitative research interviewing. Sage, Thousand Oaks, CA.

LeCompte, M.D. and Schensul, J.J. 1999. Designing and conducting ethnographic research. Ethnographer's toolkit 1. Altamira Press, Walnut Creek, CA.

Lefebvre, S., Montgomery, P., Michel, I., Warren, C., Larose, T. and Kauppi, C. 2012. The role of public health inspectors in maintaining housing in northern and rural communities: Recommendations to support public health practice. Can. J. Public Health. 103(2): 84-89. doi: $10.17269 /$ cjph.103.2907.

National Collaborating Centre for Determinants of Health. 2015. Glossary of essential health equity terms. Available at http://nccdh. ca/images/uploads/comments/English_Glossary_Nov17_FINAL.pdf. (Accessed 26 February 2016.)

Nova Scotia Department of Health and Wellness. (n.d.). Nova Scotia public health equity protocol. Halifax, NS. Available at http://novascotia.ca/dhw/publichealth/documents/05-HealthEquity-Protocol.pdf. (Accessed 26 February 2016.)

Pham, M.T., Jones, A.Q., Sargeant, J.M., Marshall, B.J. and Dewey, C.E. 2010. A qualitative exploration of the perceptions and information needs of public health inspectors responsible for food safety. BMC Public Health. 10: 345. doi: 10.1186/1471-2458-10-345.

Public Health Agency of Canada. 2008. The chief public health officer's report on the state of public health in Canada, 2008: Addressing health inequalities. Ottawa, ON. Available at http:// www.phac-aspc.gc.ca/cphorsphc-respcacsp/2008/fr-rc/pdf/CPHOReport-e.pdf. (Accessed 26 February 2016.)

Rideout, K. and National Collaborating Centre for Determinants of Health. 2015. Equity in environmental health practice: Findings of a pilot study. Vancouver, BC. Available at http://www.ncceh.ca/ documents/evidence-review/equity-environmental-health-practicefindings-pilot-study. (Accessed 26 February 2016.)

Rubin, H.J. and Rubin, I.S. 2005. Qualitative interviewing: The art of hearing data. Sage, Thousand Oaks, CA.

Stewart, J. and Bourn, C. 2013. The environmental health practitioner: New evidence-based roles in housing, public health and well-being. Perspect. Public Health. 133(6): 325-329. doi: $10.1177 / 1757913913491366$.

The Health Communication Unit. 2002. Using focus groups. Toronto, ON. Available at http://www.sswm.info/sites/default/files/reference attachments/THCU\%202002\%20Using\%20Focus\%20Group.pdf. (Accessed 26 February 2016.) 